\title{
Open science in space
}

\author{
Scientific and medical research conducted in space can bring benefits for all humankind, but this will require \\ commercial space flight companies to embrace open data principles.
}

T: he recent lift off of two billionaires on their respective private spacecraft was not quite the giant leap seen in 1969 , but has still been heralded as the start of a new golden era of space flight. Astronomers have observed the stars for millennia, but modern space research is interdisciplinary, and includes life sciences and medicine. For people on Earth to benefit from space science, data from research in space must be open, regardless of the interests of commercial companies.

Space flight is one of humanity's greatest achievements and has a unique ability to amaze and inspire future generations of mathematicians, scientists, engineers and doctors. Indeed, an increasing number of astronauts are medically or scientifically trained, including virologist Kate Rubin, astrophysicist, engineer and physician David Saint-Jacques, and Serena Auñón-Chancellor, board-certified in internal and aerospace medicine. Medical research in space has two goals: to enable people to travel safely in low Earth orbit, to the Moon, and then to Mars and back; and to improve health on Earth through discoveries made in space.

Several aspects of human biology are uniquely affected by the conditions and exposures of space travel. Microgravity, radiation and isolation each take their toll on the human body and mind. Muscle volume and bone mass both decrease during time in microgravity, with astronauts losing around $1 \%$ of their bone density each month. At $0.38 g$, the gravity on Mars may be enough to regenerate bone cells lost during the 7-month trip. If not, a ticket to Mars may be one-way, or vertebrae could be crushed during re-entry to Earth.

Cosmic rays regularly pass through astronauts, causing blinding flashes when traveling through the eye, and leading to an increased risk of cancer and cataracts. With no electromagnetic field on Mars to divert harmful radiation, people may need to live underground. The eye is also affected by microgravity, leading to far-sightedness in many astronauts.
Space science can also offer insights on aspects of human health on Earth. Everyone who has lived through lockdowns knows the mental-health effects of isolation. Space agencies have such extensive experience in dealing with isolation that they have assisted with crises on Earth, providing support, for example, to the 33 trapped Chilean miners in 2010, as well as during the COVID-19 pandemic. A voyage that goes boldly to Mars may need a ship's counselor.

Space research could also provide a model for sustainable living on Earth, such as how to deal with water shortages (Scott Kelly drank 730 litres of his own recycled sweat and urine), use of telemedicine in remote communities, and 3D printing of organs and medical supplies. The geographic information system (GIS) has been used to map the deforestation of the Amazon, track outbreaks of infectious diseases, including COVID-19, and identify remote villages for polio vaccinations. Digital image processing, which was invented to enhance pictures of the Moon, is used on images from CT and MRI scans, and forms an essential part of medical diagnostics. Vibration platforms, originally developed by the USSR for cosmonaut training, are now used to treat muscle atrophy and osteoporosis in older people

Supply trips for the International Space Station (ISS) are provided by commercial companies, as part of a growing number of public private partnerships, and bring new experiments for the astronauts. In August, the crew of the ISS received a 3D printer, cardiac muscle cells, a new $\mathrm{CO}_{2}$ removal system or 'scrubber', and some slime mold.

The cardiac muscle cells will be used to model the skeletal muscle disorder sarcopenia, which can lead to falls and functional decline, particularly in older people. Sarcopenia is also seen in astronauts, and so researchers hope that microgravity will have a similar effect on myocytes in vitro, and allow the development of a myotube model for pre-clinical drug screening. With no currently approved treatments, this would benefit people in space and on Earth.
The $\mathrm{CO}_{2}$ scrubber traps carbon dioxide from the spacecraft atmosphere in a mineral known as zeolite, with the $\mathrm{CO}_{2}$ then either vented or converted into water - technology that could be used in closed environments on Earth, as well as for removing greenhouse gases. The slime mold is in orbit for education and inspiration, as part of a French school's science project.

Any trip into space should not just inspire, but should also tangibly benefit all humanity. The principles of open science should be embraced by commercial space flight companies. Proprietary information is unavoidable, especially with regards to propulsion systems, but scientific research conducted on space stations, whether publically or privately operated, should be published and widely disseminated.

The greatest benefit will come if health data from all space tourists and astronauts are stored, with their consent, in electronic health records in a single database or trusted research environment, so that the space science community can access and analyze the data. As the number of people in space increases, this unique dataset will be an invaluable tool for further understanding and mitigating the effects of space flight on the human body. As with all health data, diversity is also key and is currently lacking, notwithstanding China and India's space ambitions, the ESA's parastronaut feasibility project, and NASA's Artemis mission. This increased diversity is late but welcome.

Scientific and medical research in space has been an exemplar of international cooperation and openness. Companies have an important role for innovation and for reducing costs, but without data sharing, important scientific advances will have a limited effect and a narrow benefit. As the first person in space, Yuri Gagarin, said, Earth is "too small for conflict and just big enough for cooperation". Cooperation, and not conflict, should also embody space science.

Published online: 14 September 2021 https://doi.org/10.1038/s41591-021-01508-1 